\title{
Ovarian steroid cell tumors, not otherwise specified: Analysis of nine cases with a literature review
}

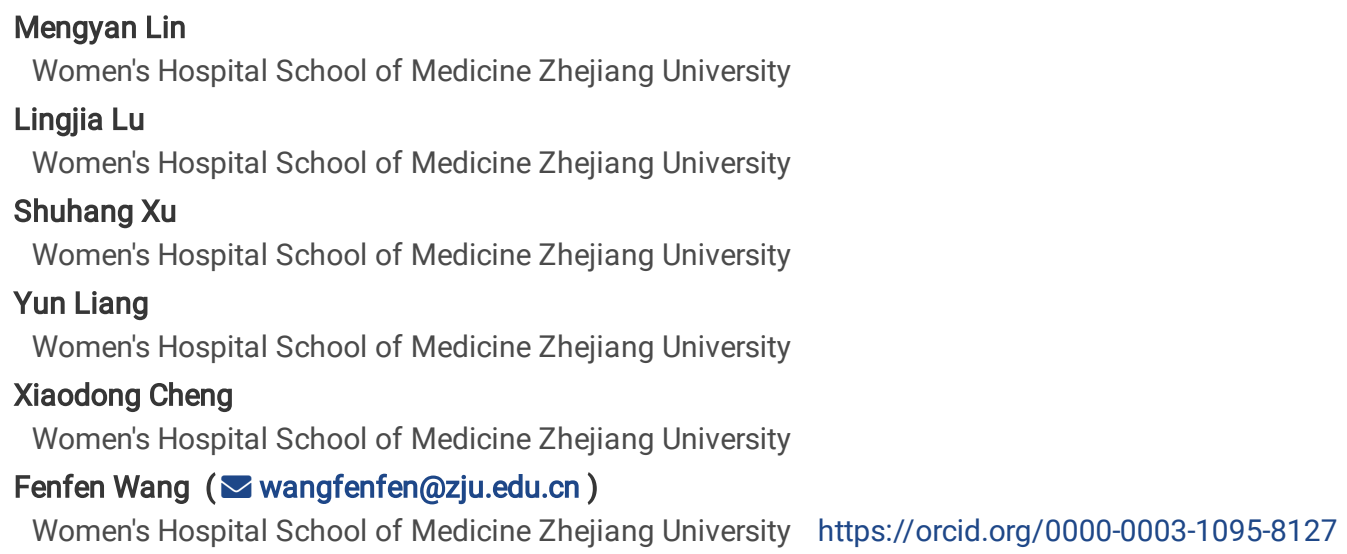

\section{Research}

Keywords: ovarian steroid cell tumors, not otherwise specified, treatment

Posted Date: September 13th, 2021

DOI: https://doi.org/10.21203/rs.3.rs-889596/v1

License: (c) (7) This work is licensed under a Creative Commons Attribution 4.0 International License. Read Full License 


\section{Abstract \\ Objective}

Ovarian steroid cell tumors (SCTs), not otherwise specified (NOS), are rare, with few large studies. The purpose of this study was to analyze the clinical features, prognosis, and treatment choices for SCT-NOS patients of different age groups.

\section{Methods}

This was a retrospective study. We identified nine SCT-NOS cases, confirmed by post-operative histopathological examination, and analyzed clinical features, surgical procedures, and follow up outcomes. We also reviewed cases reports of SCT-NOS.

\section{Results}

A total of nine cases were included. The age range was 9-68 years (mean, $41.89 \pm 19.72$ years). Clinical features included virilization, amenorrhea, abdominal pain, vaginal bleeding, isosexual precocious puberty, Cushing's syndrome, and abnormal weight gain with elevated testosterone levels. The follow up interval ranged 5-53 months and no recurrence was observed.

\section{Conclusion}

Ovarian SCTs covered all age groups, with manifestations of androgen excess. Younger patients appeared to have a more favorable prognosis, which provided more opportunities for these patients to pursue treatment options that will preserve reproductive function.

\section{Background}

Ovarian steroid cell tumors (SCTs) are rare ovarian sex cord stromal tumors that account for less than $0.1 \%$ of all ovarian tumors [1]. SCTs occur in females of all ages, causing symptoms such as virilization, isosexual precocious puberty in adolescents, Cushing's syndrome, and infertility. These clinical manifestations may result from excessive secretion of steroid hormones as well as tumor mass effects. There are three subtypes of SCT, according to cell origin: stromal luteomas, Leydig cell tumors, and not otherwise specified (NOS) tumors [1]. Approximately $60 \%$ of SCTs are of the NOS subtype and are usually benign; however, some of these tumors do have malignant behavior, as is observed with peritoneal metastases [2, 3]. Survival and fertility are affected by the clinical manifestations of SCT-NOS as well as the different treatment options. Here, we describe the clinical characteristics of nine cases of SCT-NOS and review literature reports related to this disease. The aim of this study was to summarize features for diagnosis, treatment, and prognosis of ovarian SCT-NOS and provide new clues for future treatment.

\section{Methods}

We included all cases diagnosed as ovarian SCT-NOS through histopathological examinations in the Women's Hospital School of Medicine at Zhejiang University from January 2013 to December 2020. There were 12 retrospective cases, and three of these were excluded because there was a lack of patient history, history of operations at other centers, or because patients were lost to follow up. The remaining nine cases included patients who had received surgical treatment. Clinical records, biological results, histological results, and follow-up information were analyzed for these patients.

\section{Results}

Clinical features

For the nine cases analyzed, patient age ranged from 9-68 years (mean, $41.89 \pm 19.72$ years). One patient was premenarchal, four were of reproductive age, and four were postmenopausal. Adnexal masses were the reason for operation in six cases; the other SCTs were incidentally discovered after operations related to endometrial cancers (two cases) or endometriosis (one case).

Most of the nine ovarian SCT patients experienced symptoms of endocrine or metabolic disorders. Three patients presented with virilization symptoms such as hirsutism, acne, and hoarseness; virilization symptoms were alleviated in all patients within 2-3 months. Amenorrhea was observed in two patients along with virilization symptoms. In one patient, regular menses resumed 2 months post-operation, while another amenorrhea persisted in the other patient due to administration of gonadotropin releasing hormone agonist (GnRHa) therapy. Two patients reported abdominal pain and one experienced irregular vaginal bleeding. Isosexual precocious puberty presenting as early development of breasts, occurred in one case involving an 9-year-old girl, and was alleviated postoperatively. Five patients were overweight [body mass index (BMI) $\geq 24$ $\mathrm{kg} / \mathrm{m}^{2}$ ] and two were obese (BMI $\left.\geq 28 \mathrm{~kg} / \mathrm{m}^{2}\right)$; Cushing's syndrome was observed in one obese patient (Table 1).

Page $2 / 14$ 
Table 1

Clinical features of nine patients (This table can be insert between page 3 and page 4 .)

\begin{tabular}{|c|c|c|c|c|c|c|c|c|c|}
\hline \multirow[t]{2}{*}{ No } & \multirow{2}{*}{$\begin{array}{l}\text { Age } \\
\text { (years) }\end{array}$} & \multicolumn{5}{|c|}{ Clinical manifestations } & \multirow{2}{*}{$\begin{array}{l}\text { BMI } \\
\left(\mathrm{kg} / \mathrm{m}^{2}\right)\end{array}$} & \multirow{2}{*}{$\begin{array}{l}\mathrm{T} \\
\text { (nmol/L) }\end{array}$} & \multirow{2}{*}{$\begin{array}{l}\text { DHEAS } \\
\text { (jmol/L) }\end{array}$} \\
\hline & & $\begin{array}{l}\text { Lower } \\
\text { abdominal } \\
\text { mass }\end{array}$ & $\begin{array}{l}\text { Abdominal } \\
\text { discomfort }\end{array}$ & Endocrine disorders & $\begin{array}{l}\text { Obesity/ } \\
\text { overweight }\end{array}$ & Others & & & \\
\hline 1 & 14 & + & $\begin{array}{l}\text { Abdominal } \\
\text { pain }\end{array}$ & $\begin{array}{l}\text { Amenorrhea, acne, } \\
\text { hirsutism, Cu-shing's } \\
\text { syndrome }\end{array}$ & Obesity & / & 30.11 & $3.8 \uparrow$ & $9.68 \uparrow$ \\
\hline 2 & 53 & + & - & - & - & / & 23.83 & $6.1 \uparrow$ & 6.78 \\
\hline 3 & 25 & + & - & $\begin{array}{l}\text { Amenorrhea, hirsutism, } \\
\text { acne, }\end{array}$ & Obesity & Fatty liver & 29.64 & $17.6 \uparrow$ & $8.25 \uparrow$ \\
\hline 4 & 55 & - & - & $\begin{array}{l}\text { Irregular vaginal } \\
\text { bleeding }\end{array}$ & Overweight & $\begin{array}{l}\text { Endometrial } \\
\text { cancer stage } \varangle \mathrm{C}\end{array}$ & 24.09 & 1.4 & $8.34 \uparrow$ \\
\hline 5 & 51 & + & - & Hirsutism & Overweight & / & 27.97 & - & - \\
\hline 6 & 59 & + & - & - & - & / & 19.43 & $5.2 \uparrow$ & $11.8 \uparrow$ \\
\hline 7 & 43 & + & - & - & - & $\begin{array}{l}\text { Dysmenorrhea, } \\
\text { endometriosis }\end{array}$ & 22.20 & 0.4 & 5.2 \\
\hline 8 & 68 & - & - & $\begin{array}{l}\text { Regular vaginal } \\
\text { bleeding until } 65 \text {-years- } \\
\text { old }\end{array}$ & Overweight & $\begin{array}{l}\text { Endometrial } \\
\text { cancer stage } ₫ A\end{array}$ & 24.75 & 0.9 & 6.1 \\
\hline 9 & 9 & + & $\begin{array}{l}\text { Abdominal } \\
\text { pain }\end{array}$ & $\begin{array}{l}\text { Premature mammary } \\
\text { development }\end{array}$ & - & / & 13.72 & 0.8 & - \\
\hline
\end{tabular}

Clinical symptoms typically differ based on hormonal secretion, and preoperative serum androgen levels were elevated in most patients. Five patients had abnormal hormone levels. In four patients, serum testosterone $(T$ ) levels were increased to $3.8-17.6 \mathrm{nmol} / \mathrm{L}$ (normal range, $0.3-3.0$ $\mathrm{nmol} / \mathrm{L}$ ). In four patients, preoperative serum dehydroepiandrosterone-sulfate (DHEAS) levels were elevated to $8.25-11.8 \mu \mathrm{mol} / \mathrm{L}$ (normal range, 0.96-6.95 $\mathrm{mmol} / \mathrm{L}$ ). Testosterone and DHEAS levels returned to normal postoperatively in two patients (Table 1). One menopausal patient had slightly higher estradiol as well as slightly lower follicle-stimulating hormone (FSH) and luteinizing hormone (LH) levels, according to normal menopausal levels (FSH: 34.98 IU/L, LH: $21.89 \mathrm{IU} / \mathrm{L}$, estradiol: 101.9 pmol/L).

Pelvic masses were found in seven patients. Pelvic ultrasound revealed a heterogeneous mass in five patients, along with lower blood flow resistance index (RI, range 0.31-0.52); one patient exhibited no obvious blood flow signal. Magnetic resonance imaging (MRI) of five patients indicated a T1 isointense and T2 hyperintense mass. The lesions were well enhanced after administration of gadolinium-diethylenetriamine pentaacetic acid. Although pelvic masses were present in most patients, one patient did not present with an ovarian mass preoperatively and was ultimately diagnosed by pathology.

Surgical treatment

Four patients underwent bilateral salpingo-oophorectomy with or without hysterectomy, and three young patients underwent ovarian tumor resection. Other procedures occurred in only one patient, as described in Table 2. Two cases were diagnosed with SCT-NOS based on histopathological diagnosis without visible ovarian tumors. Tumor diameter ranged from 2-5 cm in the other six cases (Table 2). Macroscopic examination revealed yellowish-brown, soft, solid masses in four cases. One case showed a mixture of cystic and solid dark red masses and the other showed a hard, solid mass. Thus, a pelvic mass was a typical sign of SCT-NOS, and surgical resection was an important treatment.

Histopathological features of eight cases are described in Table 2 (Fig. 1). Hemorrhage and nuclear atypia grade $₫-\otimes$ were discovered in a 68 -yearold patient with endometrial cancer. Nuclear atypia grade $\nabla-\varangle$ was observed alone in a 9-year-old patient. In these cases, neither necrosis nor two or more mitotic cells per 10 high power fields were found.

Follow up

Follow-up time for the nine cases ranged from 5-53 months. Disease recurrence had not occurred in any of the cases recently (Table 2). 
Table 2

Surgical procedures and follow-up of nine patients

\begin{tabular}{|c|c|c|c|c|c|c|c|c|c|}
\hline \multirow[t]{2}{*}{ No } & \multirow{2}{*}{$\begin{array}{l}\text { Age } \\
\text { (years) }\end{array}$} & \multirow{2}{*}{$\begin{array}{l}\text { Surgical } \\
\text { procedure }\end{array}$} & \multirow{2}{*}{$\begin{array}{l}\text { Tumor } \\
\text { size } \\
(\geq 7 \mathrm{~cm})\end{array}$} & \multicolumn{4}{|c|}{ Pathological features } & \multirow{2}{*}{$\begin{array}{l}\text { Follow- } \\
\text { up } \\
\text { (months) }\end{array}$} & \multirow[t]{2}{*}{ Recurrence } \\
\hline & & & & Necrosis & Hemorrhage & $\begin{array}{l}\text { Mitosis ( } \geq 2 \text { per } \\
10 \mathrm{HPF} \text { ) }\end{array}$ & 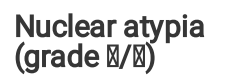 & & \\
\hline 1 & 14 & OTR & - & - & - & - & - & 5 & No \\
\hline 2 & 53 & RSO & - & - & - & - & - & 9 & No \\
\hline 3 & 25 & OTR & - & - & - & - & - & 11 & No \\
\hline 4 & 55 & $\begin{array}{l}\text { HE, BSO, OE, } \\
\text { PLND }\end{array}$ & - & - & - & - & - & 12 & No \\
\hline 5 & 51 & HE, BSO & I & - & - & - & - & 27 & No \\
\hline 6 & 59 & BSO & - & - & - & - & - & 28 & No \\
\hline 7 & 43 & HE, BS, OER & - & - & - & - & - & 32 & No \\
\hline 8 & 68 & EHE, BSO & - & - & + & - & + & 46 & No \\
\hline 9 & 9 & OTR & - & - & - & - & + & 53 & No \\
\hline
\end{tabular}

\section{Discussion}

Ovarian SCT-NOS have not been studied across a large number of cases, except for Hayes and Scully's investigation of 63 cases [1]. We searched PubMed, EBSCO, OVID, and Web of Science for cases of SCT-NOS in English with the search string (("steroid cell tumor" OR "steroid cell neoplasm") AND (ovary OR ovaries OR ovarian)) AND ("not otherwise specified" OR "nos"). A total of 329 articles were found, of which 129 were duplicates, 117 were irrelevant, 10 were non-English, and five contained incomplete information. These unmatched articles were excluded and 12 other case reports were added through references. Ultimately, we found 80 articles describing 95 cases of SCT-NOS. We excluded 39 of these cases due to lack of follow-up information or follow-up periods that were too short (less than 3 months), which left 56 cases (Table 3 ). We did not include the information from the 63 cases in Hayes and Scully's article because details were lacking in each of these cases. 
Table 3

Clinical features of 65 cases reported (This table can be insert between page 6 and page 7.)

\begin{tabular}{|c|c|c|c|c|c|c|c|c|c|c|}
\hline \multirow[t]{2}{*}{ Case } & \multirow[t]{2}{*}{ First author } & \multirow{2}{*}{$\begin{array}{l}\text { Age } \\
\text { (years) }\end{array}$} & \multirow{2}{*}{$\begin{array}{l}\text { Surgical } \\
\text { procedure }\end{array}$} & \multirow{2}{*}{$\begin{array}{l}\text { Tumor } \\
\text { diameter } \\
\text { (cm) }\end{array}$} & \multicolumn{4}{|c|}{ Pathological features } & \multirow{2}{*}{$\begin{array}{l}\text { Adjuvant } \\
\text { therapy }\end{array}$} & \multirow{2}{*}{$\begin{array}{l}\text { Follow-up } \\
\text { (months) }\end{array}$} \\
\hline & & & & & Necrosis & Hemorrhage & $\begin{array}{l}\text { Mitosis } \\
(\geq 2 \\
\text { per } 10 \\
\text { HPF })\end{array}$ & $\begin{array}{l}\text { Nuclear } \\
\text { atypia } \\
\text { (grade } \\
\bigotimes / 囚)\end{array}$ & & \\
\hline 1 & Haroon [9] & 3 & LSO & 7 & - & - & - & - & - & $\begin{array}{l}\text { No } \\
\text { recurrence } \\
(25)\end{array}$ \\
\hline 2 & $\begin{array}{l}\text { Yoshimatsu } \\
\text { [10] }\end{array}$ & 4 & RSO & 8 & + & + & $+(3)$ & - & $B E P \times 3$ & $\begin{array}{l}\text { No } \\
\text { recurrence } \\
(7)\end{array}$ \\
\hline 3 & Qian [11] & 5 & RSO & 8 & - & - & - & - & - & $\begin{array}{l}\text { No } \\
\text { recurrence } \\
(60)\end{array}$ \\
\hline 4 & Gupta [12] & 5 & $\begin{array}{l}\text { Tumor } \\
\text { resection }\end{array}$ & NA & NA & NA & NA & NA & - & $\begin{array}{l}\text { No } \\
\text { recurrence } \\
\text { (4) }\end{array}$ \\
\hline 5 & $\begin{array}{l}\text { Sawathiparnich } \\
\text { [13] }\end{array}$ & 6 & LSO & 7 & + & - & $\begin{array}{l}+(2- \\
5)\end{array}$ & - & - & $\begin{array}{l}\text { No } \\
\text { recurrence } \\
(6)\end{array}$ \\
\hline 6 & Lee [14] & 8 & RO & 5 & - & - & - & - & - & $\begin{array}{l}\text { No } \\
\text { recurrence } \\
(16)\end{array}$ \\
\hline 7 & Bas [6] & 9 & HE, BSO & 2.5 & - & - & - & - & Glucocorticoid & $\begin{array}{l}\text { No } \\
\text { recurrence } \\
(52)\end{array}$ \\
\hline 8 & Yilmaz [4] & 13 & $\begin{array}{l}\text { Tumor } \\
\text { resection }\end{array}$ & 2.5 & - & - & - & - & - & $\begin{array}{l}\text { No } \\
\text { recurrence } \\
(6)\end{array}$ \\
\hline 9 & Schnuckle [15] & 14 & LSO & 3.2 & - & + & - & - & - & $\begin{array}{l}\text { No } \\
\text { recurrence } \\
(24)\end{array}$ \\
\hline 10 & Yokozawa [5] & 16 & $\begin{array}{l}\text { Tumor } \\
\text { resection }\end{array}$ & 4 & - & - & - & - & - & $\begin{array}{l}\text { No } \\
\text { recurrence } \\
(15)\end{array}$ \\
\hline 11 & Yuan [16] & 17 & $\begin{array}{l}\text { Tumor } \\
\text { resection }\end{array}$ & 8 & - & - & - & - & - & $\begin{array}{l}\text { No } \\
\text { recurrence } \\
(6)\end{array}$ \\
\hline 12 & Sielert [17] & 20 & $\mathrm{RO}$ & 5.5 & - & - & - & - & - & $\begin{array}{l}\text { No } \\
\text { recurrence } \\
(18)\end{array}$ \\
\hline 13 & Jiang [18] & 21 & $\begin{array}{l}\mathrm{HE}, \mathrm{BSO}, \\
\mathrm{OE}\end{array}$ & $\begin{array}{l}\text { L 20, } \\
\text { R } 15\end{array}$ & + & + & $+(\rrbracket 10)$ & + & $\mathrm{PVB} \times 4$ & $\begin{array}{l}\text { Continued } \\
\text { and died } \\
\text { (10) }\end{array}$ \\
\hline 14 & Santo [19] & 21 & RSO & 11.5 & - & + & $+(4)$ & - & - & $\begin{array}{l}\text { No } \\
\text { recurrence } \\
(43)\end{array}$ \\
\hline 15 & Zhang [20] & 21 & LSO, OE & 5.8 & - & - & - & - & - & $\begin{array}{l}\text { No } \\
\text { recurrence } \\
(3)\end{array}$ \\
\hline 16 & $\begin{array}{l}\text { Matsukawa } \\
\text { [21] }\end{array}$ & 22 & $\begin{array}{l}\text { Tumor } \\
\text { resection }\end{array}$ & 3 & - & - & - & - & - & $\begin{array}{l}\text { No } \\
\text { recurrence } \\
(60)\end{array}$ \\
\hline 17 & Arora [22] & 22 & HE, LSO & 2 & NA & NA & NA & NA & - & $\begin{array}{l}\text { Recurred } \\
\text { (108) }\end{array}$ \\
\hline
\end{tabular}

LSO: left salpingo-oophorectomy; RSO: right salpingo-oophorectomy; BSO: bilateral salpingo-oophorectomy; LO: left oophorectomy; RO: right oophorectomy; BO: bilateral oophorectomy; HE: hysterectomy; OE: omentectomy; PLND: pelvic lymph node dissection; PLNS: pelvic lymph node sampling; AE: appendectomy; TD: tumor debulking; NA: not available; BEP: bleomycin + etoposide + cisplatin; PVB: cisplatin + vincristine + bleomycin 


\begin{tabular}{|c|c|c|c|c|c|c|c|c|c|c|}
\hline \multirow[t]{2}{*}{ Case } & \multirow[t]{2}{*}{ First author } & \multirow{2}{*}{$\begin{array}{l}\text { Age } \\
\text { (years) }\end{array}$} & \multirow{2}{*}{$\begin{array}{l}\text { Surgical } \\
\text { procedure }\end{array}$} & \multirow{2}{*}{$\begin{array}{l}\text { Tumor } \\
\text { diameter } \\
\text { (cm) }\end{array}$} & \multicolumn{4}{|c|}{ Pathological features } & \multirow{2}{*}{$\begin{array}{l}\text { Adjuvant } \\
\text { therapy }\end{array}$} & \multirow{2}{*}{$\begin{array}{l}\text { Follow-up } \\
\text { (months) }\end{array}$} \\
\hline & & & & & Necrosis & Hemorrhage & $\begin{array}{l}\text { Mitosis } \\
(\geq 2 \\
\text { per } 10 \\
\text { HPF })\end{array}$ & $\begin{array}{l}\text { Nuclear } \\
\text { atypia } \\
\text { (grade } \\
\text { ख/区) }\end{array}$ & & \\
\hline 18 & Jiang [18] & 23 & $\begin{array}{l}\text { Tumor } \\
\text { resection }\end{array}$ & 6 & No & - & - & - & - & $\begin{array}{l}\text { No } \\
\text { recurrence } \\
\text { (36) }\end{array}$ \\
\hline 19 & Wong [23] & 24 & RSO & 4.5 & - & - & - & - & - & $\begin{array}{l}\text { No } \\
\text { recurrence } \\
\text { (9) }\end{array}$ \\
\hline 20 & Haroon [9] & 26 & RSO & 4.5 & - & - & - & - & - & $\begin{array}{l}\text { No } \\
\text { recurrence } \\
\text { (15) }\end{array}$ \\
\hline 21 & Menghua [24] & 28 & LO & 2.5 & + & NA & $\begin{array}{l}\text { Active } \\
\text { cell } \\
\text { growth }\end{array}$ & NA & - & $\begin{array}{l}\text { Recurred } \\
(42), \text { died } \\
(58)\end{array}$ \\
\hline 22 & Swain [25] & 28 & LSO & 5.9 & NA & NA & NA & NA & - & $\begin{array}{l}\text { No } \\
\text { recurrence } \\
\text { (12) }\end{array}$ \\
\hline 23 & Haroon [9] & 28 & HE, BSO & $3 \& 2.5$ & - & No & $+(2)$ & - & - & $\begin{array}{l}\text { No } \\
\text { recurrence } \\
\text { (49) }\end{array}$ \\
\hline 24 & $\mathrm{Li}[26]$ & 29 & RSO & 6 & + & + & + & + & $\begin{array}{l}\text { Docetaxel + } \\
\text { Ne-daplatin } \\
\times 8\end{array}$ & $\begin{array}{l}\text { Recurred } \\
(18), \text { died } \\
(24)\end{array}$ \\
\hline 25 & Tai [7] & 29 & RSO & 10 & - & + & - & - & - & $\begin{array}{l}\text { No } \\
\text { recurrence } \\
\text { (24) }\end{array}$ \\
\hline 26 & Alves [3] & 30 & $\begin{array}{l}\text { Tumor } \\
\text { resection }\end{array}$ & 2 & - & - & - & - & - & $\begin{array}{l}\text { No } \\
\text { recurrence } \\
\text { (36) }\end{array}$ \\
\hline 27 & Liu [27] & 30 & $\begin{array}{l}\text { Tumor } \\
\text { resection }\end{array}$ & 3 & - & - & - & - & - & $\begin{array}{l}\text { No } \\
\text { recurrence } \\
\text { (12) }\end{array}$ \\
\hline 28 & Haroon [9] & 32 & HE, BSO & 4 & - & - & - & - & - & $\begin{array}{l}\text { No } \\
\text { recurrence } \\
\text { (71) }\end{array}$ \\
\hline 29 & Chung [28] & 35 & $\begin{array}{l}\text { HE, BSO, } \\
\text { PLND, OE, } \\
\text { AE }\end{array}$ & 4.9 & - & - & - & - & $\mathrm{GnRHa} \times 6$ & $\begin{array}{l}\text { No } \\
\text { recurrence } \\
\text { (43) }\end{array}$ \\
\hline 30 & Stephens [29] & 35 & RSO & 5 & NA & NA & - & NA & - & $\begin{array}{l}\text { No } \\
\text { recurrence } \\
\text { (6) }\end{array}$ \\
\hline 31 & Faten [8] & 36 & RO & 4.5 & - & - & - & - & - & $\begin{array}{l}\text { No } \\
\text { recurrence } \\
\text { (12) }\end{array}$ \\
\hline 32 & Haroon [9] & 37 & LSO & 7 & - & - & - & - & - & $\begin{array}{l}\text { No } \\
\text { recurrence } \\
\text { (23) }\end{array}$ \\
\hline 33 & Ben [30] & 39 & LSO & 3.4 & NA & NA & NA & NA & - & $\begin{array}{l}\text { No } \\
\text { recurrence } \\
\text { (8) }\end{array}$ \\
\hline 34 & Chen [31] & 40 & RSO & 6 & NA & NA & NA & NA & - & $\begin{array}{l}\text { No } \\
\text { recurrence } \\
\text { (36) }\end{array}$ \\
\hline
\end{tabular}

LSO: left salpingo-oophorectomy; RSO: right salpingo-oophorectomy; BSO: bilateral salpingo-oophorectomy; LO: left oophorectomy; RO: right oophorectomy; BO: bilateral oophorectomy; HE: hysterectomy; OE: omentectomy; PLND: pelvic lymph node dissection; PLNS: pelvic lymph node sampling; AE: appendectomy; TD: tumor debulking; NA: not available; BEP: bleomycin + etoposide + cisplatin; PVB: cisplatin + vincristine + bleomycin 


\begin{tabular}{|c|c|c|c|c|c|c|c|c|c|c|}
\hline \multirow[t]{2}{*}{ Case } & \multirow[t]{2}{*}{ First author } & \multirow{2}{*}{$\begin{array}{l}\text { Age } \\
\text { (years) }\end{array}$} & \multirow{2}{*}{$\begin{array}{l}\text { Surgical } \\
\text { procedure }\end{array}$} & \multirow{2}{*}{$\begin{array}{l}\text { Tumor } \\
\text { diameter } \\
\text { (cm) }\end{array}$} & \multicolumn{4}{|c|}{ Pathological features } & \multirow{2}{*}{$\begin{array}{l}\text { Adjuvant } \\
\text { therapy }\end{array}$} & \multirow{2}{*}{$\begin{array}{l}\text { Follow-up } \\
\text { (months) }\end{array}$} \\
\hline & & & & & Necrosis & Hemorrhage & $\begin{array}{l}\text { Mitosis } \\
(\geq 2 \\
\text { per } 10 \\
\text { HPF })\end{array}$ & $\begin{array}{l}\text { Nuclear } \\
\text { atypia } \\
\text { (grade } \\
\text { (囚/囚) }\end{array}$ & & \\
\hline 35 & Varras [32] & 40 & $\mathrm{HE}, \mathrm{BSO}$ & 6.8 & - & - & - & - & - & $\begin{array}{l}\text { No } \\
\text { recurrence } \\
(18)\end{array}$ \\
\hline 36 & Haroon [9] & 43 & RSO & 10 & - & - & $+(5)$ & + & - & $\begin{array}{l}\text { Recurred } \\
(34)\end{array}$ \\
\hline 37 & Zang [33] & 46 & LSO & 12 & NA & NA & NA & - & - & $\begin{array}{l}\text { No } \\
\text { recurrence } \\
(12)\end{array}$ \\
\hline 38 & Kim [34] & 46 & $\begin{array}{l}\text { HE, BSO, } \\
\text { PLND, OE }\end{array}$ & 7.7 & NA & NA & NA & NA & - & $\begin{array}{l}\text { Recurred } \\
(60)\end{array}$ \\
\hline 39 & Reedy [35] & 46 & $\begin{array}{l}\text { HE, LSO, } \\
\text { PLNS, OE }\end{array}$ & 3.6 & - & - & - & - & - & $\begin{array}{l}\text { No } \\
\text { recurrence } \\
(12)\end{array}$ \\
\hline 40 & Lee [36] & 47 & $\begin{array}{l}\text { HE, BSO, } \\
\text { OE }\end{array}$ & 11 & - & + & $+(5)$ & + & $\mathrm{BEP} \times 3$ & $\begin{array}{l}\text { No } \\
\text { recurrence } \\
(24)\end{array}$ \\
\hline 41 & Wang [37] & 48 & $\begin{array}{l}\text { HE, RSO, } \\
\text { PLND, OE }\end{array}$ & 4 & - & - & - & + & - & $\begin{array}{l}\text { No } \\
\text { recurrence } \\
(6)\end{array}$ \\
\hline 42 & Nakasone [38] & 50 & $\begin{array}{l}\text { HE, BSO, } \\
\text { PLNS }\end{array}$ & 9 & - & - & NA & - & - & $\begin{array}{l}\text { Recurred } \\
\text { (70) }\end{array}$ \\
\hline 43 & Haroon [9] & 50 & RSO & 7 & - & - & - & - & - & $\begin{array}{l}\text { No } \\
\text { recurrence } \\
\text { (11) }\end{array}$ \\
\hline 44 & Haroon [9] & 51 & $\begin{array}{l}\text { HE, BSO, } \\
\text { PLND, OE }\end{array}$ & 13 & + & + & $+(8)$ & + & - & $\begin{array}{l}\text { Recurred } \\
\text { and died } \\
(8)\end{array}$ \\
\hline 45 & Chun [39] & 52 & LSO & 6 & - & - & $+(5)$ & - & - & $\begin{array}{l}\text { No } \\
\text { recurrence } \\
(21)\end{array}$ \\
\hline 46 & Kim [40] & 52 & $\begin{array}{l}\text { BSO, } \\
\text { PLNS, OE }\end{array}$ & 7.5 & - & - & - & - & - & $\begin{array}{l}\text { No } \\
\text { recurrence } \\
(24)\end{array}$ \\
\hline 47 & Haroon [9] & 52 & LSO & 9 & - & - & $+(4)$ & + & $\begin{array}{l}\text { PVB } \times 4 \& \\
\text { radioth-erapy }\end{array}$ & $\begin{array}{l}\text { No } \\
\text { recurrence } \\
\text { (43) }\end{array}$ \\
\hline 48 & Cooray [41] & 54 & HE, BSO & L 3, R 4 & - & - & - & - & - & $\begin{array}{l}\text { No } \\
\text { recurrence } \\
(12)\end{array}$ \\
\hline 49 & Faraj [42] & 55 & BO & 4.5 & NA & NA & NA & NA & - & $\begin{array}{l}\text { No } \\
\text { recurrence } \\
(12)\end{array}$ \\
\hline 50 & Layla [43] & 65 & BSO, TD & 7 & - & NA & $+(2)$ & - & $\mathrm{BEP} \times 6$ & $\begin{array}{l}\text { Continued } \\
\text { (18) }\end{array}$ \\
\hline 51 & Brewer [44] & 58 & $\begin{array}{l}\text { HE, BSO, } \\
\text { TD }\end{array}$ & 10 & NA & NA & $+(9)$ & + & $\begin{array}{l}\text { BEP } \times 2 \\
\text { GnRHa }\end{array}$ & $\begin{array}{l}\text { Recurred } \\
(28)\end{array}$ \\
\hline 52 & Sedhom [45] & 67 & $\begin{array}{l}\text { HE, BSO, } \\
\text { TD }\end{array}$ & 9.8 & NA & + & $+(22)$ & NA & - & $\begin{array}{l}\text { Died after } \\
\text { operati-on }\end{array}$ \\
\hline 53 & Stephens [46] & 67 & HE, BSO & 1.2 & - & - & - & - & - & $\begin{array}{l}\text { No } \\
\text { recurrence } \\
\text { (3) }\end{array}$ \\
\hline
\end{tabular}




\begin{tabular}{|c|c|c|c|c|c|c|c|c|c|c|}
\hline \multirow[t]{2}{*}{ Case } & \multirow[t]{2}{*}{ First author } & \multirow{2}{*}{$\begin{array}{l}\text { Age } \\
\text { (years) }\end{array}$} & \multirow{2}{*}{$\begin{array}{l}\text { Surgical } \\
\text { procedure }\end{array}$} & \multirow{2}{*}{$\begin{array}{l}\text { Tumor } \\
\text { diameter } \\
\text { (cm) }\end{array}$} & \multicolumn{4}{|c|}{ Pathological features } & \multirow{2}{*}{$\begin{array}{l}\text { Adjuvant } \\
\text { therapy }\end{array}$} & \multirow{2}{*}{$\begin{array}{l}\text { Follow-up } \\
\text { (months) }\end{array}$} \\
\hline & & & & & Necrosis & Hemorrhage & $\begin{array}{l}\text { Mitosis } \\
(\geq 2 \\
\text { per } 10 \\
\text { HPF) }\end{array}$ & $\begin{array}{l}\text { Nuclear } \\
\text { atypia } \\
\text { (grade } \\
\text { 囚/囚) }\end{array}$ & & \\
\hline 54 & Haroon [9] & 67 & BSO & 3.4 & - & - & - & - & - & $\begin{array}{l}\text { No } \\
\text { recurrence } \\
\text { (27) }\end{array}$ \\
\hline 55 & Singh [47] & 70 & RO & 5 & NA & NA & NA & NA & - & $\begin{array}{l}\text { No } \\
\text { recurrence } \\
\text { (5) }\end{array}$ \\
\hline 56 & Powell [48] & 93 & $\begin{array}{l}\text { HE, BSO, } \\
\text { sigmoid- } \\
\text { ectomy, } \\
\text { colostomy }\end{array}$ & 21 & + & NA & + & + & - & $\begin{array}{l}\text { Recurred } \\
\text { and multi } \\
\text { metastasis } \\
(10)\end{array}$ \\
\hline \multicolumn{11}{|c|}{$\begin{array}{l}\text { LSO: left salpingo-oophorectomy; RSO: right salpingo-oophorectomy; BSO: bilateral salpingo-oophorectomy; LO: left oophorectomy; RO: right } \\
\text { oophorectomy; BO: bilateral oophorectomy; HE: : hysterectomy; OE: omentectomy; FLND: pelvic lymph node dissection; PLNS: pelvic lymph } \\
\text { node sampling; AE: appendectomy; TD: tumor debulking; NA: not available; BEP: bleomycin + etoposide + cisplatin; PVB: cisplatin + vincristine + } \\
\text { bleomycin }\end{array}$} \\
\hline
\end{tabular}

Of the 56 cases, the age span of SCT-NOS patients was 3-93 years (median, 33.5 years). Excessive weight gain and virilization features such as hirsutism, acne, baldness, hoarseness, clitoromegaly, along with elevated testosterone were common at any age. For patients of reproductive age, amenorrhea was persistent because of hormonal parasecretion. For premenarchal girls, isosexual precocious puberty and advanced growth affected most. In our cases, virilization manifestations such as hirsutism, acne, and amenorrhea, as well as excessive weight gain were also quite common due to testosterone elevation. Isosexual precocious puberty occurred in one nine-year-old patient from our set of cases, and presented as premature mammary development. Other less common manifestations such as hypertension, abdominal pain, vaginal bleeding, Cushing's syndrome, and acanthosis nigricans were also reported in the cases analyzed in this study. The symptoms caused by SCT-NOS vanished within 116 months post-operation, among the cases with no recurrence. However, Yilmaz and Yokozawa discovered that some changes, including hoarseness, were irreversible even after hormone secretion abnormalities were corrected by tumor elimination [4,5]. Whether grown adolescent height and weight were normalized could not be determined due to lack of follow-up data. A previous study by Bas reported that a 9-year-old SCTNOS patient had coexistent congenital adrenal hyperplasia due to classic 11 beta-hydroxylase deficiency, and the patient had initial hypertension that progressed and showed hypertensive retinopathy 2 years post-operation [6]. Alves, Faten, and Tai each published cases involving infertility with follow-up periods of 36 months, 12 months, and 24 months, respectively; none of the cases reported successful pregnancy [3, 7, 8]. Data related to infertility were limited due to lack of long-term follow-up. Some menopausal cases exhibited elevated estradiol as well as low LH and FSH levels, which may have induced endometrial disease from persistent estradiol influence. In our cases, two postmenopausal patients also presented with endometrial adenocarcinoma. Although reproductive hormone levels corresponded with postmenopausal levels in both patients, one also experienced vaginal bleeding until age 65 year. It is suspicious that long-term, persistent estradiol stimulation resulted in vaginal bleeding symptoms, and this suggests the potential presence of STC-NOS along with induced endometrial adenocarcinoma.

Approximately 25-43\% SCTs-NOS have malignant potential [1]. The previous study by Hayes and Scully pointed out five pathological features that were correlated with malignancy potential: 1$)$ two or more mitotic fields per 10 high power fields ( $92 \%$ malignant); 2 ) necrosis ( $86 \%$ malignant); 3 ) tumor diameter $\geq 7 \mathrm{~cm}$ (78\% malignant); 4) hemorrhage (77\% malignant); and 5) grade II-III nuclear atypia (64\% malignant) [1]. Among the 65 cases searched, with the exception of eight cases with missing pathological details, approximately half (28/57) presented with at least one malignant feature. Metastasis was observed in seven cases with primary operation, and at least two malignant pathological features were reported in six cases. Another case was reported according to only one feature. Two other cases showed capsule and vascular infiltration or vascular growth, respectively, and both presented with at least two features. In patients 40 years or younger, the rate of malignant pathological features was $44.83 \%(13 / 29)$, and the rate for patients over 40 years of age was $78.95 \%(15 / 19)(P \otimes 0.05, \chi 2$ test). Metastasis, infiltration, and vascular growth occurred in $10.34 \%(3 / 29)$ of the patients 40 years or younger and in $31.58 \%(6 / 19)$ of the patients over 40 (P区0.05, $\chi 2$ test). It seems that SCTs-NOS in patients over the age of 40 are more likely to have malignant behavior. In our cases, a 68 -year-old patient experienced

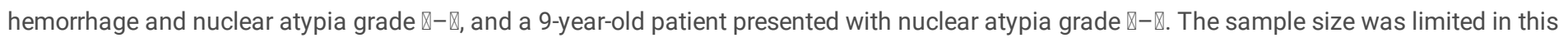
study, and larger scale clinical studies comparing different ages are still needed to provide more conclusive evidence. 
Table 4

Clinical features, treatments, and outcomes of recurred or progressed cases

\begin{tabular}{|c|c|c|c|c|}
\hline Case & $\begin{array}{l}\text { Age } \\
\text { (years) }\end{array}$ & Clinical features & Second therapy & $\begin{array}{l}\text { Outcome } \\
\text { (months)* }\end{array}$ \\
\hline 13 & 21 & $\begin{array}{l}\text { Virilization, amenorrhea, ascites, acanthosis nigricans, initial } \\
\text { surgery delayed } 4 \text { years }\end{array}$ & $\mathrm{PVB} \times 4$ & $\begin{array}{l}\text { Continued and died } \\
\text { (10) }\end{array}$ \\
\hline 17 & 22 & $\begin{array}{l}\text { Virilization, amenorrhea, with hereditary leiomyomatosis and } \\
\text { renal cell carcinoma syndrome }\end{array}$ & RSO, RPLND & Not mentioned \\
\hline 21 & 28 & $\begin{array}{l}\text { Virilization, Cushing's syndrome, hypertension, irregular } \\
\text { menstruation }\end{array}$ & Metastatic lesions resection & Recurred (12), died (16) \\
\hline 24 & 29 & Lower back and leg pain, normal reproductive hormone & Not mentioned & Died (6) \\
\hline 38 & 46 & Ascites & $\begin{array}{l}\text { Metastatic lesions resection, } \\
\text { BEP }\end{array}$ & Tumor free (9) \\
\hline 42 & 50 & Virilization & $\begin{array}{l}\mathrm{BEP} \times 3, \mathrm{TP} \times 8 \text {, chemotherapy } \\
\text { invalid, GnRHa }\end{array}$ & $\begin{array}{l}\text { No symptom (22 after } \\
\text { first GnRHa) }\end{array}$ \\
\hline 44 & 51 & Lower abdominal pain, vaginal spotting, anorexia & Not mentioned & Died \\
\hline 51 & 58 & Virilization, pelvic discomfort & Increased dose of $\mathrm{GnRHa}$ & Not mentioned \\
\hline 50 & 65 & Virilization, abdominal pain, ascites, bilateral lower limb edema & $\mathrm{BEP} \times 3$ & $\begin{array}{l}\text { Continued (18), lost to } \\
\text { follow up }\end{array}$ \\
\hline 56 & 93 & $\begin{array}{l}\text { Virilization, abdominal pain, bilateral lower limb edema, } \\
\text { aggressive and violent behavior }\end{array}$ & $\begin{array}{l}\text { GnRHa \& weekly paclitaxel } \\
\text { for } 1 \text { month }\end{array}$ & $\begin{array}{l}\text { Refused therapies and } \\
\text { progressed }\end{array}$ \\
\hline
\end{tabular}

*The interval time after recurrence or progress; PVB: cisplatin + vincristine + bleomycin; BEP: bleomycin + etoposide + cisplatin; TP: paclitaxel + carboplatin; RSO: right salpingo-oophorectomy; RPLND: right pelvic lymph node dissection.

Disease recurrence or progression occurred in 10 cases (17.86\%) (Table 4). The tumor-free interval ranged from 0-108 months, with a median time of 23 months. The age of recurrence ranged from 21-93 years, and none of patients younger than 20 years of age reported disease recurrence or progression. The recurrence rate was $11.43 \%$ (4/35) for patients aged 40 years or younger, $28.57 \%(6 / 21)$ for those older than 40 years. In the group above 40 years of age, malignant behavior and disease recurrence were more likely to happen. Ascites was a unique manifestation for this group and may serve as an indicator of recurrence following initial therapy. One case that recurred did not report any malignant pathological features, with the longest tumor-free interval of 9 years. Six cases displayed at least two malignant pathological features, whereas three cases displayed only one. The tumor-free interval increased considerably as the feature numbers decreased (Fig. 2).

The reported treatments for disease recurrence or progression in SCT-NOS were tumor excision operations, chemotherapy, and GnRHa therapy. Two recurrent cases received surgery alone, with one reporting 12 months tumor free and a survival time of 16 months. In two reported cases, tumor progression showed no response to chemotherapy alone. Another relapsed case did not respond to chemotherapy but recovered after shifting to GnRHa therapy, and remained tumor free for 22 months. This suggested that for relapsed SCT-NOS patients, GnRHa therapy may be an appropriate choice for adjuvant treatment. Brewer reported a case with aggravated symptoms as well as computed tomography (CT) showing enlarged lymph nodes and uronephrosis during chemotherapy alone after the primary operation; however, the symptoms faded, lymph nodes were diminished, and uronephrosis was improved after switching from chemotherapy to $\mathrm{GnRHa}$. This again indicated an important role for GnRHa as an SCT-NOS adjuvant treatment.

Tumor resections alone were performed in eight patients over 40-years-old with no reported disease recurrence. Unilateral salpingo-oophorectomies or oophorectomies were performed in 20 patients over 40, and there were two reports of disease recurrence. These surgeries helped to preserve ovarian function and fertility for young patients with an occurrence rate of $7.14 \%$, which was lower than the overall rate. As discussed above, the malignancy potential and occurrence appeared to be lower in people younger than 40 years and, therefore, surgeries that preserve fertility and ovarian function can be considered when obvious malignant features are absent.

\section{Conclusions}

Due to the low incidence of the SCT-NOS, it was difficult to conduct large-scale clinical cohort studies and there was a lack of consistent recommendations for treatment. SCT-NOS are a rare ovarian tumor subtype that can occur at any age. Virilization, weight gain, and amenorrhea with elevated testosterone secretion are common among SCT-NOS patients. Isosexual precocious puberty can also occur in immature girls. Though endocrine abnormalities often recover after surgery, the resulting development effects remain. Therefore, it is important to improve the early diagnosis of this disease to reduce abnormal development caused by endocrine factors as well as relieve anxiety and psychological pressure in younger patients. For older patients, SCT-NOS has malignant potential, and it is important to properly complete pathological assessment 
because the risk of malignancy and the recurrence rate both increase after age of 40 years. It is of the upmost importance for clinicians to pay careful attention to this phenomenon and effectively manage it. Younger patients have a more favorable prognosis, which provides the chance to consider fertility and ovarian function preserving surgeries.

This study included mostly cases published in public journals, with necessary complete information and prognostic outcomes, between 1991 and 2020. This relatively complete retrospective clinical study provides effective information and value for the diagnosis and treatment of SCT-NOS.

\section{Abbreviations}

SCT: ovarian steroid cell tumor

NOS: not otherwise specified

GnRHa: gonadotropin releasing hormone agonist

BMI: body mass index

T: testosterone

DHEAS: dehydroepiandrosterone-sulfate

FSH: follicle-stimulating hormone

LH: luteinizing hormone

$\mathrm{RI}$ : resistance index

MRI: magnetic resonance imaging

OTR: ovarian tumor resection

RSO: right salpingo-oophorectomy

LSO: left salpingo-oophorectomy

BSO: bilateral salpingo-oophorectomy

BS: bilateral salpingectomy

HE: hysterectomy

EHE: extrafascial hysterectomy

OE: omentectomy

PLND: pelvic lymph node dissection

RPLND: right pelvic lymph node dissection

PLNS: pelvic lymph node sampling

OER: ovarian endometriotic cyst resection

LO: left oophorectomy

RO: right oophorectomy

BO: bilateral oophorectomy

$\mathrm{AE}$ : appendectomy

TD: tumor debulking

NA: not available

BEP: bleomycin + etoposide + cisplatin 
PVB: cisplatin + vincristine + bleomycin

TP: paclitaxel + carboplatin

CT: computed tomography

\section{Declarations}

Ethics approval and consent to participate

Study was reviewed and approved by Ethics Committee of Women's Hospital, School of Medicine, Zhejiang University (approval number IRB20210092-R).

\section{Consent for publication}

Not applicable.

\section{Availability of data and materials}

The datasets used and analysed during the current study are available from the corresponding author on reasonable request.

\section{Competing interests}

The authors declare that they have no competing interests.

\section{Funding}

None.

\section{Authors' contributions}

MYL and FFW designed the study, analyzed the patients' data, searched literatures, and drafted the manuscript. LJL and SHX participated in collecting the patients' data and searching literatures. XDC reviewed the final manuscript. YL checked the histological examination of the steroid cell tumors. All authors read and approved the final manuscript.

\section{Acknowledgements}

This work was supported by the Zhejiang Provincial Natural Science Foundation of China (grant number LY21H160032), and the Key research and development program of Zhejiang province (grant number 2019C03010).

\section{Authors' information}

${ }^{1}$ Department of Gynecological Oncology, Women's Hospital, School of Medicine, Zhejiang University, No.1 Xueshi Road, Hangzhou City, Zhejiang Province, 310006, China. ${ }^{2}$ Department of Pathology, Women's Hospital, School of Medicine, Zhejiang University, No.1 Xueshi Road, Hangzhou City, Zhejiang Province, 310006, China.

\section{References}

1. Hayes MC, Scully RE. Ovarian steroid cell tumors (not otherwise specified):a clinicopathological analysis of 63 cases. Am J Surg Pathol. 1987;11:835-45.

2. Tan EC, Khong CC, Bhutia K. A rare case of steroid cell tumor, not otherwise specified (NOS), of the Ovary in a Young Woman. Case Rep Obstet Gynecol. 2019;2019:4375839.

3. Alves P, Sa I, Brito M, Carnide C, Moutinho O. An early diagnosis of an ovarian steroid cell tumor not otherwise specified in a woman. Case Rep Obstet Gynecol. 2019;2019:2537480-0.

4. Yılmaz-Ağladioğlu S, Savaş-Erdeve Ş, Boduroğlu E, Önder A, Karaman İ, Çetinkaya S, Aycan Z. A girl with steroid cell ovarian tumor misdiagnosed as non-classical congenital adrenal hyperplasia. Turk J Pediatr. 2013;55(4):443-6.

5. Yokozawa T, Asano R, Nakamura T, Furuya M, Nagashima Y, Koyama-Sato M, Kanda Y, Hirahara F, Sakakibara H. Steroid cell tumour, not otherwise specified: Rare case with primary amenorrhoea in a 16-year-old. J Obstet Gynaecol. 2015;35(8):867-8.

6. Bas F, Saka N, Darendeliler F, Tuzlali S, Ilhan R, Bundak R, Gunoz H. Bilateral ovarian steroid cell tumor in congenital adrenal hyperplasia due to classic 11 beta-hydroxylase deficiency. J Pediatr Endocrinol Metab. 2000;13(6):663-7. 
7. Tai YJ, Chang WC, Kuo KT, Sheu BC. Ovarian steroid cell tumor, not otherwise specified, with virilization symptoms. Taiwan J Obstet Gynecol. 2014;53(2):260-2.

8. Faten H, Dorra G, Slim C, Wajdi S, Nadia C, Kais C, Tahia B, Mohamed A. Ovarian steroid cell tumor (not otherwise specified): a case report of ovarian hyperandrogenism. Case Rep Oncol Med. 2020;2020:6970823.

9. Haroon S, Idrees R, Fatima S, Memon A, Kayani N. Ovarian steroid cell tumor, not otherwise specified: a clinicopathological and immunohistochemical experience of 12 cases. J Obstet Gynaecol Res. 2015;41(3):424-31.

10. Yoshimatsu T, Nagai K, Miyawaki R, Moritani K, Ohkubo K, Kuwabara J, Tatsuta K, Kurata M, Fukushima M, Kitazawa R, et al. Malignant ovarian steroid cell tumor, not otherwise specified, causes virilization in a 4-year-old girl: a case report and literature review. Case Rep Oncol. 2020;13(1):358-64.

11. Qian L, Shen Z, Zhang X, Wu D, Zhou Y. Ovarian steroid cell tumor, not otherwise specified: a case report and literature review. Mol Clin Oncol. 2016;5(6):839-41.

12. Gupta P, Goyal S, Gonzalez-Mendoza LE, Noviski N, Vezmar M, Brathwaite CD, Misra M. Corticotropin-independent cushing syndrome in a child with an ovarian tumor misdiagnosed as nonclassic congenital adrenal hyperplasia. Endocr Pract. 2008;14(7):875-9.

13. Sawathiparnich P, Sitthinamsuwan P, Sanpakit K, Laohapensang M, Chuangsuwanich T. Cushing's syndrome caused by an ACTH-producing ovarian steroid cell tumor, NOS, in a prepubertal girl. Endocrine. 2009;35(2):132-5.

14. Lee SH, Kang MS, Lee GS, Chung WY. Refractory hypertension and isosexual pseudoprecocious puberty associated with renin-secreting ovarian steroid cell tumor in a girl. J Korean Med Sci. 2011;26(6):836-8.

15. Schnuckle EM, Williamson A, Carpentieri D, Taylor S. Ovarian sex cord stromal tumor, steroid cell, NOS in an adolescent: a case report. J Pediatr Adolesc Gynecol. 2020;34(1):94-7.

16. Yuan X, Sun Y, Jin Y, Chen X, Wang X, Ji T, Wang C, Dai H. Ovarian steroid cell tumor, not otherwise specified, treated with surgery: a case report and review of literature. Int J Clin Exp Pathol. 2019;12(4):1434-8.

17. Sielert L, Liu C, Nagarathinam R, Craig L. Androgen-producing steroid cell ovarian tumor in a young woman and subsequent spontaneous pregnancy. J Assist Reprod Genet. 2013;30(9):1157-60.

18. Jiang W, Tao X, Fang F, Zhang S, Xu C. Benign and malignant ovarian steroid cell tumors, not otherwise specified: case studies, comparison, and review of the literature. J Ovarian Res. 2013;6:53.

19. Santo RE, Sabino T, Agapito A. Ovarian steroid cell tumor not otherwise specified with virilizing manifestations: case report. Acta Obstétrica e Ginecológica Portuguesa. 2016;10(4):336-9.

20. Zhang X, Lue B. Ovarian steroid cell tumor, not otherwise specified (NOS): an unusual case with myelolipoma. Int J Gynecol Pathol. 2011;30(5):460-5.

21. Matsukawa J, Takahashi T, Hada Y, Kameda W, Ota K, Fukase M, Takahashi K, Matsuo K, Mizunuma H, Nagase S. Successful laparoscopic resection of virilizing ovarian steroid cell tumor, not otherwise specified, in a 22-year-old woman: a case report and evaluation of the steroidogenic pathway. Fukushima J Med Sci. 2019;65(3):133-9.

22. Arora RMD, Eble JNMD, Pierce HHP, Crispen PLMD, Desimone CPMD, Lee EYMD, Karabakhtsian RGMD. Bilateral ovarian steroid cell tumors and massive macronodular adrenocortical disease in a patient with hereditary leiomyomatosis and renal cell cancer syndrome. Am $\mathrm{J}$ Clin Pathol. 2012;138(suppl_1):A203.

23. Wong FCK, Chan AZ, Wong WS, Kwan AHW, Law TSM, Chung JPW, Kwok JSS, Chan AOK. Hyperandrogenism, elevated 17hydroxyprogesterone and its urinary metabolites in a young woman with ovarian steroid cell tumor, not otherwise specified: case report and review of the literature. Case Rep Endocrinol. 2019;2019:9237459.

24. Menghua Y, Mingcai Q, Mei Z. Symptomatic Cushing syndrome and hyperandrogenemia revealing steroid cell ovarian neoplasm with late intra-abdominal metastasis. BMC endocrine disorders. 2014;14(1):59-72.

25. Swain J, Sharma S, Prakash V, Agrawal NK, Singh SK. Steroid cell tumor: a rare cause of hirsutism in a female. Endocrinol Diabetes Metab Case Rep. 2013;2013:130030.

26. Li K, Zhu F, Xiong J, Liu F. A rare occurrence of a malignant ovarian steroid cell tumor not otherwise specified: a case report and literature review. Oncol Lett. 2014;8(2):770-4.

27. Liu A-x, Sun J, Shao W-q. Jin H-m, Song W-q: Steroid cell tumors, not otherwise specified (NOS), in an accessory ovary: a case report and literature review. Gynecol Oncol. 2005;97(1):260-2.

28. Chung D-H, Lee S-H, Lee K-B. A case of ovarian steroid cell tumor, not otherwise specified, treated with surgery and gonadotropin releasing hormone agonist. J Menopausal Med. 2014;20(1):39-42.

29. Stephens JW, Fielding A, Verdaguer R, Freites O. A steroid-cell tumor of the ovary resulting in massive androgen excess early in the gonadol steroidogenic pathway. Gynecol Endocrinol. 2008;24(3):151-3.

30. Ben Haj Hassine MA, Msakni I, Siala H, Rachdi R. Laparoscopic management of an ovarian steroid cell tumor, not otherwise specified causing virilization and amenorrhea: a case report. Case Rep Clin Pathol. 2015;3(1):10-3.

Page $12 / 14$ 
31. Chen S, Li R, Zhang X, Lu L, Li J, Pan H, Zhu H. Combined ovarian and adrenal venous sampling in the localization of adrenocorticotropic hormone-independent ectopic Cushing syndrome. J Clin Endocrinol Metab. 2018;103(3):803-8.

32. Varras M, Vasilakaki T, Skafida E, Akrivis C. Clinical, ultrasonographic, computed tomography and histopathological manifestations of ovarian steroid cell tumour, not otherwise specified: our experience of a rare case with female virilisation and review of the literature. Gynecol Endocrinol. 2011;27(6):412-8.

33. Zang L, Ye M, Yang G, Li J, Liu M, Du J, Gu W, Jin N, Yang L, Ba J, et al. Accessory ovarian steroid cell tumor producing testosterone and cortisol: a case report. Medicine. 2017;96(37):e7998.

34. Kim JS, Park SN, Kim BR. Recurrent ovarian steroid cell tumor, not otherwise specified managed with debulking surgery, radiofrequency ablation, and adjuvant chemotherapy. Obstet Gynecol Sci. 2014;57(6):534-8.

35. Reedy MB, Richards WE, Ueland F, Uy K, Lee EY, Bryant C, van Nagell JR Jr. Ovarian steroid cell tumors, not otherwise specified: a case report and literature review. Gynecol Oncol. 1999;75(2):293-7.

36. Lee J, John VS, Liang SX, D'Agostino CA, Menzin AW. Metastatic malignant ovarian steroid cell tumor: a case report and review of the literature. Case Rep Obstet Gynecol. 2016;2016:6184573.

37. Wang PH, Chao HT, Lee RC, Lai CR, Lee WL, Kwok CF, Yuan CC, Ng HT. Steroid cell tumors of the ovary: clinical, ultrasonic, and MRI diagnosis a case report. Eur J Radiol. 1998;26(3):269-73.

38. Nakasone T, Nakamoto T, Matsuzaki A, Nakagami H, Aoki Y. Direct evidence on the efficacy of GnRH agonist in recurrent steroid cell tumor-not otherwise specified. Gynecol Oncol Rep. 2019;29:73-5.

39. Chun YJ, Choi HJ, Lee HN, Cho S, Choi JH. An asymptomatic ovarian steroid cell tumor with complete cystic morphology: a case report. Obstet Gynecol Sci. 2013;56(1):50-5.

40. Kim YT, Kim SW, Yoon BS, Kim SH, Kim JH, Kim JW, Cho NH. An ovarian steroid cell tumor causing virilization and massive ascites. Yonsei Med J. 2007;48(1):142-6.

41. Cooray SMA, Bulugahapitiya UDS, Samarasinghe K, Samarathunga P. Steroid cell tumor not otherwise specified of bilateral ovaries: a rare cause of post menopausal virilization. Indian J Endocrinol Metab. 2013;17(Suppl 1):262-4.

42. Faraj G, Di Gregorio S, Misiunas A, Faure EN, Villabrile P, Stringa I, Petroff N, Bur G. Virilizing ovarian tumor of cell tumor type not otherwise specified: a case report. Gynecol Endocrinol. 1998;12(5):347-52.

43. Abdullah LS. Ovarian steroid cell tumor, NOS presenting with massive ascites and elevated CA-125. JKAU Med Sci. 2011;18(3):107-14.

44. Brewer CA, Shevlin D. Encouraging response of an advanced steroid-cell tumor to GnRH agonist therapy. Obstet Gynecol. 1998;92(4 Pt 2):661-3.

45. Sedhom R, Hu S, Ohri A, Infantino D, Lubitz S. Symptomatic Cushing's syndrome and hyperandrogenemia in a steroid cell ovarian neoplasm: a case report. J Med Case Rep. 2016;10(1):278.

46. Stephens JW, Katz JR, McDermott N, MacLean AB, Bouloux PM. An unusual steroid-producing ovarian tumour: case report. Hum Reprod. 2002;17(6):1468-71.

47. Singh P, Deleon F, Anderson R. Steroid cell ovarian neoplasm, not otherwise specified: a case report and review of the literature. Case Rep Obstet Gynecol. 2012;2012:253152.

48. Powell JL, Dulaney DP, Shiro BC. Androgen-secreting steroid cell tumor of the ovary. South Med J. 2000;93(12):1201-4.

\section{Figures}




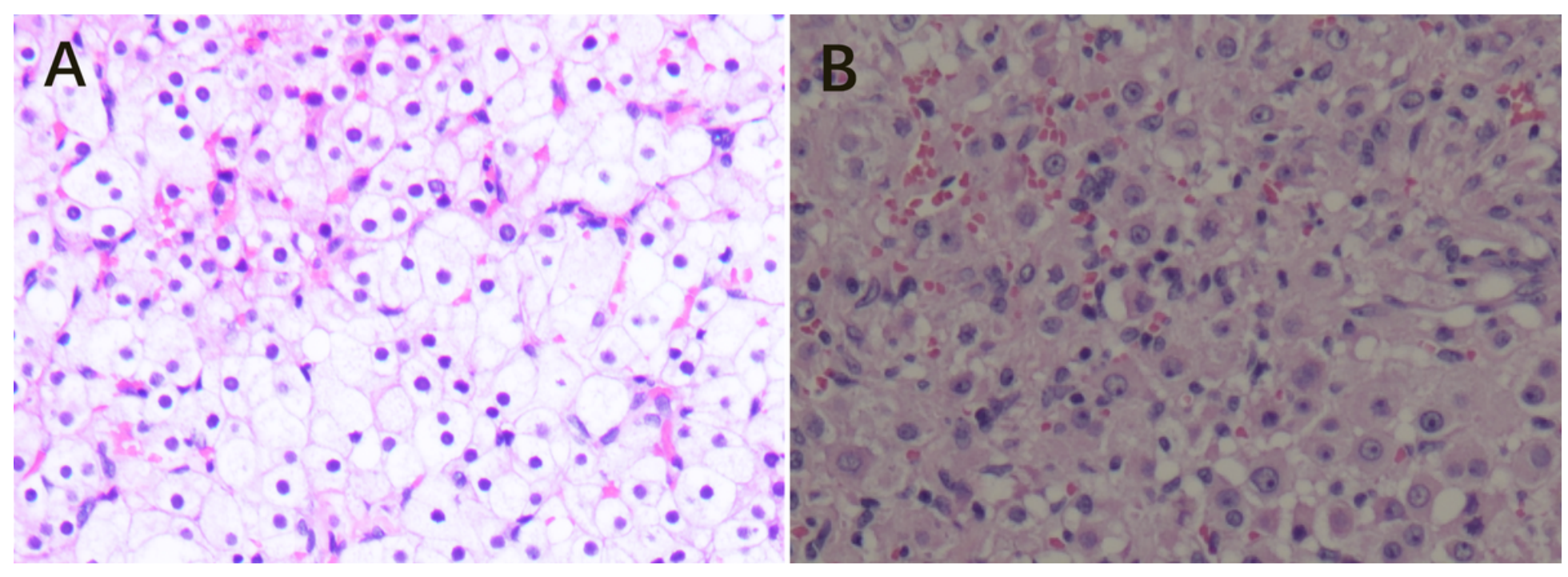

Figure 1

Microscopic appearance of steroid cell tumor, not otherwise specified $₫$ H\&E囚. (A) Benign SCT-NOS cells with abundant cytoplasm (20x). (B) SCTNOS cells with nuclear atypia grade $\nabla-\otimes(20 x)$.

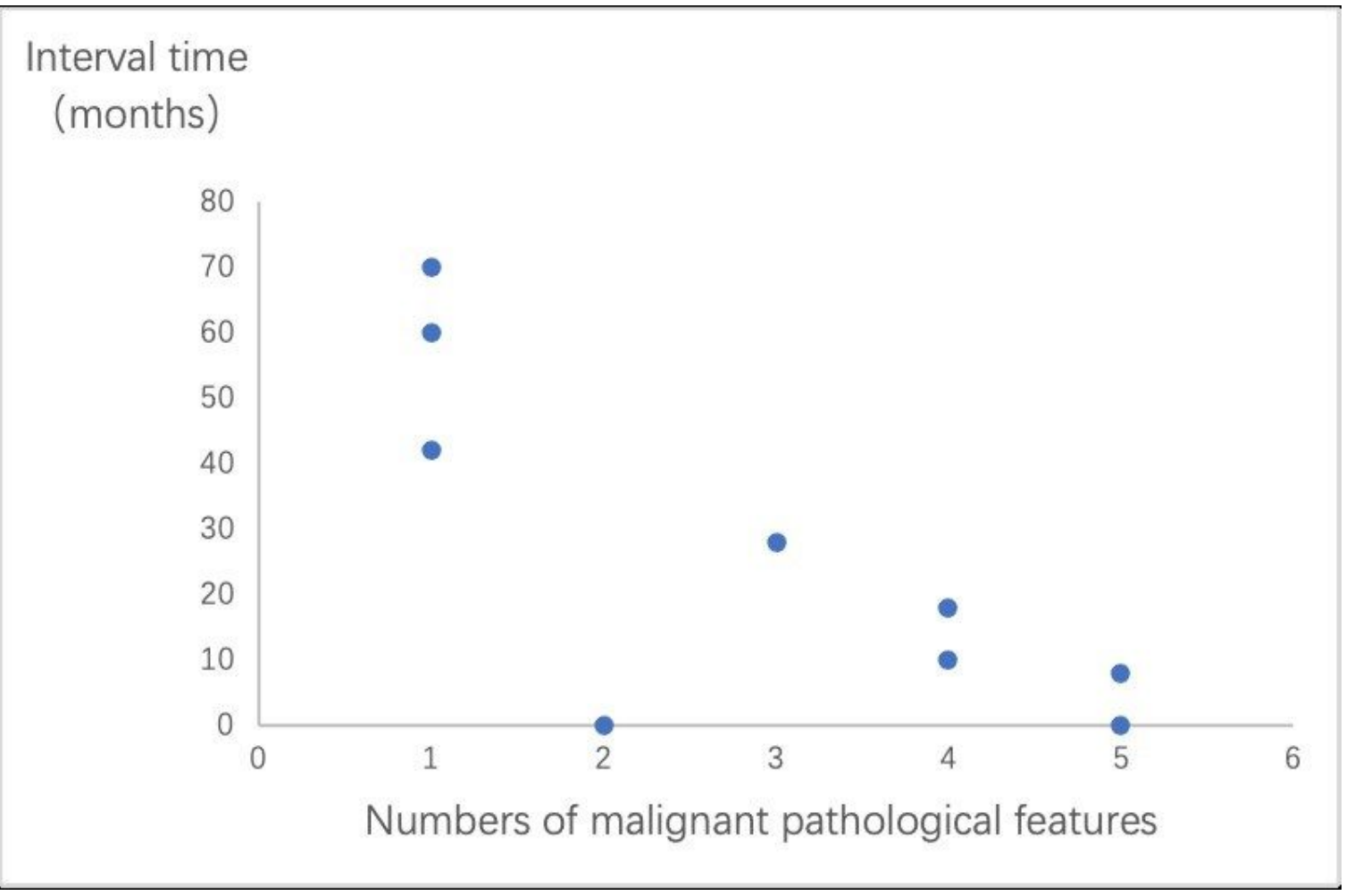

\section{Figure 2}

Relationship between number of malignant pathological features and interval time. 